\title{
ETHICS AND DISASTERS IN THE WORK OF ALBERT SCHWEITZER
}

\author{
KATARÍNA KOMENSKÁ
}

\begin{abstract}
Traditional ethical frameworks are challenged in disaster settings as they are often too rigorous to be applied to such situations. Nonetheless, the role of moral theories in discussions on disasters should not be dismissed. Indeed, some of the ideas and concepts in traditional ethical frameworks and moral theories may be a source of inspiration in such debates. Therefore, the present paper presents the two main concepts in Albert Schweitzer's philosophical thinking: the concept of cultural crisis and his understanding of ethics. These concepts form the basis of Schweitzer's formulation of an ethics of the reverence for life as an answer to the cultural crisis and the need for a new ethics for a modern, humane civilisation. His thinking is reflected through the scope of disaster ethics and its potential to enrich discussions on disaster ethics is critically analysed.
\end{abstract}

Key words: disaster ethics; culture/civilisation; virtue; reverence for life.

\section{Introduction}

Ethics and philosophy have always attempted to reflect on the problems people face in their lives. Despite some of these issues having belonged to human history from its very beginning, it is only recently that they have been subjected to a systematic ethical consideration. An important motive for extending the scope of ethics in this way is the development of applied ethics; in other words, the aspiration of ethics to practically reflect on issues related to the life of society and its members. One issue that falls within the scope of modern ethics is the ethical reflection of disasters and catastrophes: disaster ethics. This has only recently become an independent discipline within ethics and it seeks to ethically reevaluate and analyse disasters and consider how we might ethically respond to them. Disaster ethics is concerned with all issues related to moral decision-making and actions taken within the context of disasters. The distinctive characteristic of disaster ethics is found in the specific challenges presented by disasters and the way in which they differ from everyday situations. ${ }^{1}$

\footnotetext{
${ }^{1}$ Following the leading authors on disaster ethics and disaster relief management, I understand there to be three distinctive features of disaster ethics. First of all, disasters negatively impact on the life of individual beings and society. They go beyond the boundaries of the personal lives of individuals and 
Disasters are unpredictable, acute situations which cause extensive damage, destruction, and suffering, and which often cannot be dealt with by the local community on its own. The nature of these conditions is not limited only to the disaster itself (in the sense that they occur) but to the whole cycle of disaster and its individual phases. ${ }^{2}$

The people responding to disasters (victims, health care personnel, humanitarian aid workers or military personnel) often record disillusionment and a kind of ethical frustration with making ethical decisions during disasters. This is because their long-established, traditional frameworks of ethics crumble in such situations. ${ }^{3}$ Chiara Lepora is sceptical about whether it is possible to use traditional ethical frameworks, and the arguments and axiological perspectives contained within them, in ethical decision making in a disaster context. ${ }^{4}$ This can be seen not only in the professional conduct but in the decision making of all the people affected by disasters. Lepora further develops her argument and discusses the need for ethical compromises in situations where no decision is the right one. She explains the morality of compromises as follows:

Its lynchpin will be to construe compromise as a joint action, in particular a joint wrong doing - taking part in, and sharing responsibility for, the doing of things that are wrong from the point of view of those who are the parties to the compromise (Lepora, 2012, p. 1).

\section{Virtue ethics in a disaster setting?}

Despite this "negative" view of the role of moral theories, the question still stands: Can we leave aside moral theories and other theoretical concepts from the discussion on disaster ethics? Is there no useful, practical way in which ethical theories could be applied to disasters and to our decision making (either professional or personal) in such situations? Despite the limitations of applying traditional and (rather) rigid moral theories to disaster settings, ethics and its theoretical concepts should not be obeyed. What has to be taken into consideration is that they should not be applied as in formal terms but in a functional and practical sense. Since there are specific challenges to be considered in disaster ethics, there is a need to

social life. Disasters do not follow boundaries (either official or non-official) and therefore require a global perspective. Secondly, disasters are acute and unstable. The different phases in a disaster change very rapidly as do the acceptable ethical decisions. What is morally justifiable at one stage (e.g. walking past an injured man while searching for your child) can become morally unacceptable within a day or two. Thirdly, despite the fact that disaster ethics analyses morally conflicted situations commonly found in the everyday life and practice of individuals and society, they occur in different, more dramatic contexts (e.g. lies/truth, altruism/egoism, to steal/not to steal). Therefore, disasters are difficult to reassess ethically, are more complex and not transparent (in terms of motives and consequences).

${ }^{2}$ The circle of disasters and its individual phases is described in Disasters \& Emergencies produced by the World Health Organisation (WHO, 2002).

${ }^{3}$ The first attempts to develop an ethical framework for medical and humanitarian personnel in a disaster context began in the 1970s. The main objective was to produce a value- neutral and effective tool for the decision making undertaken by health care professionals attending disasters (Leaning \& Guha-Sapir, 2013).

${ }^{4}$ This is mainly because a deontological understanding of patient autonomy and dignity prevails. 
come up with a new approach to ethics; one that is more striking, holistic, and takes into consideration specific situations and cultural and global contexts. Disaster ethics should help us find a new approach which would enable us to detect and emphasize the positive characteristics of the various traditional moral theories and apply them appropriately to a disaster context.

Thus, this paper introduces some of the most important concepts in Albert Schweitzer ${ }^{5}$ understanding of ethics. Having helped revive virtue ethics in the first half of the $20^{\text {th }}$ century, Schweitzer contributed to debates on the role of ethics in the contemporary world. His thinking, critically reflected through the scope of disaster ethics, may also help us demonstrate the role virtue ethics can play in discussions on disaster ethics.

According to Rosalind Hursthouse, virtue ethics has strengths which deontological and consequential moral theories lack as they are too deeply concerned with principles, rules, and duties. Virtue ethics, on the other hand, is capable of overcoming this problem as it focuses on the virtuous action of the moral agent rather than on the ethical arguments and whether the right principle or duty is followed. In situations like disasters (where ethical principles fail to work), this characteristic of virtue ethics could become an important tool for ethical decision making and for evaluating actions resulting from disasters (Hursthouse, 1995, pp. 57-59). This paper will also highlight virtuous action and the virtuous moral agent.

\section{The crisis in contemporary culture as a man-made disaster}

Schweitzer does not directly refer to disaster ethics in his work. Nonetheless, his work does contain references to war conflicts, technological risks, and other man-made disasters. In his later years in particular, he wrote extensively on these issues. He based his arguments on a comprehensive critique of culture. ${ }^{6}$ He was strongly concerned with the negative impact humans have on the stability of life (mostly via the misuse of technology) and with the spiritual emptiness of modern civilisation.

In his work he generally defines culture/civilisation ${ }^{7}$ as the material and spiritual development of individuals and humanity as a whole (Schweitzer, 1923, p. 33) designed to ease the struggle for life. In general, culture can be accomplished in two ways: as the control of reason over nature and as the control of reason over human ideas. Both are spiritual activities as they are founded in human spirituality and the spiritual development of human beings; nevertheless, they differ in significance and importance. Schweitzer claimed that control of reason over human ideas is more important for the positive development of life. Controlling natural forces is not "pure" development as it can often turn towards "non-

\footnotetext{
${ }^{5}$ Albert Schweitzer was a famous moral philosopher, musician, theologian, humanist, and Nobel Prize winner. He was a physician and he established a hospital in Lambaréné (Gabon, formerly French Equatorial Africa) in 1913, where he worked until his death. More information about his life and philosophical background can be found in Cicovacki (2009) and Funk (2000).

${ }^{6}$ One example is his Nobel Prize Lecture entitled Peace (Schweitzer, 1954); although he had previously formulated a complex critique in his philosophical work Civilisation and Ethics (Schweitzer, 1923).

${ }^{7}$ Schweitzer does not really distinguish between these two terms and he often uses them interchangeably.
} 
culture" (something like the immoral realization of control over natural powers). Only control over human ideas is guarantee that people and nations will not use their power (established and gained by the control of natural forces) against other people's lives (Schweitzer, 1923).

What exactly does this shift towards a non-culture mean? Culture has two tendencies that can be understood as the material and the ethical development of a culture. While exerting control over natural forces can support material, technological, and scientific progress, it can also lead us to misuse and destroy them. An example, to which Schweitzer often refers, is the discovery of the nucleus of the atom and atomic research (early $20^{\text {th }}$ century). Just a few decades later, this research had become a tool of destruction and was associated with the misuse of political power by world leaders. Schweitzer concludes that without responsibility and ethical principles (in other words, without the ethical development of culture), mere knowledge of nuclear power will not lead civilisation to adopt a stable life and humanism. Therefore, material progress has to be reflected in the control over human ideas: by the ethical development of humans.

To summarize, for Schweitzer, the biggest problem in modern civilisation is its tendency to focus only on material and technological development. He even goes so far as to call this a "disastrous" aspect of humankind. He writes: "The disastrous feature of our civilization is that it is far more developed materially than spiritually" (Schweitzer, 1923, p. 38). Spiritual and ethical development is, according to Schweitzer, on the decline and is almost absent in our culture. He explains that our culture has become a culture predicated on scientific, technological, and artistic outcomes which can live without ethics or is happy to work with a minimum ethical level (Schweitzer, 1923), and that material progress has become a culture on its own.

This has not only resulted in the spiritual and moral crisis of our times, but it is also a common cause of the many man-made or conflict-based disasters. Schweitzer, without saying so directly and intentionally, is telling us that we humans could avoid or prevent many disasters. Many authors may agree with Schweitzer's argument. ${ }^{8}$ For example, Luegenbiehl explains that the complexities of disasters cannot clearly exclude human error or its effects as the cause of any catastrophe. No single cause can be determined. The complexity of disasters can therefore be used as an example to teach people more about global issues and the complexity of the modern world (Luegenbiehl, 2007). As to the need to look for the cause of disasters, he also claims that "there is enough blame to go around" and it is better to seek to prevent similar future disasters (Luegenbiehl, 2007, p. 11).

This is an inspirational idea and a starting point for discussions on "preventing" disasters. Schweitzer offers us a key turning point in disaster prevention: bring ethics back into human decision making (performed by civilians, professionals, scientists, and politicians). For Schweitzer, only ethics can protect us from man-made disasters: an ethics which formulates an essential universal ethical ideal necessary for the survival and development of the humankind. Albert Schweitzer formulated such an ethical ideal in the virtue of reverence for life.

But preventing disasters is not enough. It does not help us respond to disastrous situations once they have occurred. Cultural and moral crisis may well be the cause of many disasters

\footnotetext{
${ }^{8}$ See also Etkin \& Leman Stefanovic, 2005; O’Mathúna, 2014; Leaning \& Guha-Sapir, 2013.
} 
and we should try to "remove" this cause, but humankind will nevertheless face disasters and catastrophes (either natural or man-made). Therefore a moral theory, if it is to be practical and useful, must offer guidance (not necessarily formal or institutionalized) on how to respond to and reflect on situations like these. Before we can seek out this guidance in Schweitzer's thinking, we must undertake a deeper analysis of his understanding of ethics.

\section{Schweitzer's understanding of ethics}

There are several basic points in Schweitzer's understanding of ethics. Firstly, ethics cannot be based on institutionalized norms. Spirituality must form the basis of ethics and institutions (in any form), and not vice versa. Institutions should not have such important status as is ascribed to them nowadays (Kraus, 1944, p. 29). Kraus further explains that Schweitzer believes modern culture is over-organized and mechanical, but ethics (as Schweitzer understands them) cannot work like this (Kraus, 1944, p. 29). Ethics and its principles must be natural and discovered in all lives. It must confirm life and develop it. ${ }^{9}$ This positive attitude of ethics towards the world should be natural and complex (Schweitzer, 1923, p. 17) and not planned or organized from "above". All ethical decisions and actions should be of this nature.

Secondly, ethics must interconnect the emotional and the rational, religion and philosophy, and beliefs and knowledge. Schweitzer understands the role of reason in ethics but he still claims that reason and critical analysis may lead us only to this form of truth, which human reason is capable of depicting. What he tries to explain is that knowledge should not substitute thinking. Thinking is the only complex process of cognition capable of interrelating knowledge, desires, and emotions, and ethics must be built upon this (and not only on rational knowledge). It must become an internal part of ethical decision making and the action of man, because "knowledge on its own is nothing, intellectualism is nothing without ethical will" (Kraus, 1944, p. 66).

Therefore, according to Schweitzer, it is necessary to bring (besides intellectualism) a sort of mysticism back into ethics. ${ }^{10}$ An ethics based purely on intellectualism and knowledge is simply the external ethics of a society and its institutions, a mechanical system for managing and directing the activities of individuals. Such an ethics can never become the individual's way of thinking about the world and life. This is not a defence of irrationalism but an acquisition of the real mysticism involved in the process of rational consideration and experiencing life. Erich Fromm had this to say about this aspect of Schweitzer's ethics:

The experience of union, with man, or religiously speaking, with God, is by no means irrational. On the contrary, it is as Albert Schweitzer has pointed out, the consequence of rationalism, its most daring and radical consequence. It is based on our knowledge of the fundamental, and not accidental, limitations of our knowledge. It is the knowledge that we

\footnotetext{
${ }^{9}$ Schweitzer adopted this kind of positive attitude towards the world from Indian philosophy.

${ }^{10}$ Mysticism in the sense of a strong belief in life and its reverence. In Schweitzer's work, this mysticism is often referred to as a form of religion and as a strong basis for ethics. This mysticism is built upon love and adoration for all living beings, for nature, and for everything created by God. This belief should lead man to dedicate his life to virtuous action to develop life further.
} 
shall never "grasp" the secret of man and of the universe, but that we can know, nevertheless, in the act of love" (Fromm, 1956, pp. 32).

Through this mysticism, man understands his interconnection with the life and the need to act ethically - even if he cannot explain or understand how.

The third characteristic of Schweitzer ethics is based on an understanding of the individual and his/her role in the world. For Schweitzer, only an individual can enact ethics. Individuals create their own cathedrals or altars. They do not need institutions to build them or to experience their own lives (Cousin, 1960, p. 194). In Schweitzer's understanding ethics is strictly individualistic. This is also connected to an extremely individualistic understanding of responsibility. Responsibility defines a person's role in life (not in an individualistic sense but in a holistic one). This is a strong feature of Schweitzer's ethics, and one in which he emphasises the need to extend the circle of responsibility. This directly relates to the ethical principle of reverence for life, but its role is most significant as the idea of the moral development of moral being; as development which should never end. The aspiration to extend one's circle of moral concern is connected with the development of man's skills to reflect the world, to their individualism (ethical) and their acceptance of an ethical responsibility for all life (Bergel, 1946).

Another feature of Schweitzer's understanding of ethics is the need for a basic moral principle which would be, on one hand, universal and absolute so it could become a part of life, thinking about life, and the ethics of every human being. On the other hand, this moral principle should not place external boundaries or burdens on a person's life. This universal moral principle, empowered by the mysticism of ethics, should support man's virtuous action and moral development.

The last, and from my point of view, the most important feature of this understanding of ethics is human activity. Man's activity should be built upon ethical ideals (on the virtue of reverence for life) and therefore should be understood as a moral action. Ethics should always inform man's activity, his intention to do good. It cannot remain within the sphere of knowledge or a theoretical understanding of the concept of good. The importance of activity was famously formulated in Schweitzer's statement: My life is my argument. This statement is not only Schweitzer's attempt to show it is possible to live by such a demanding ethics, but it is also an insistence that we should cease talking and reasoning about ethics and instead live ethical lives.

\section{Difficulties of applying ethics to a disaster setting}

To conclude: Schweitzer does not understand ethics as a theoretical, academic discipline but rather as the relationship between the theoretical and the practical experience of life. Ethics must reflect on the relationship between the rational and the spiritual. It should not be built upon institutional norms but on man realizing his role in the world, his relationship towards the world, and his responsibility for his actions. The universal ethical principle cannot be dictated from outside but should be internalized by every acting agent and implemented in the decision making processes and actions. But how can we apply ethics understood in this way to issues of disaster ethics? Can any of its features be used in discussions on disasterrelated decision making processes and actions? 
There are several problems that arise when we apply Schweitzer's ethics to disaster settings. The most significant is the rejection of external norms. Schweitzer expects man to know what is good (virtuous) in a particular situation because he will always follow the main virtue of reverence for life. External norms are therefore unnecessary and unnatural. This might prove rather difficult in times of disaster as disasters are very complex, chaotic, and unpredictable. On one hand, individuals may face ethical dilemmas which are too complicated for them to be able to make ethical decisions on their own that would lead to virtuous action. Some dilemmas may even lead to situations in which none of the possible ethical decisions could be considered virtuous. In these situations, people are pushed into making ethical compromises which may negatively affect their virtuousness (e.g. stealing food to feed their child, lying to protect their possessions).

There is also a discernible conflict here between the professional and personal choices made by health care professionals, humanitarian aid workers, members of emergency and disaster relief teams, and other professionals involved in disaster relief. All professionals are tied by their professional goals and norms but, for Schweitzer, they must be humans first; humans with their own moral standards. Professional norms need not always correspond to the personal values and norms of the professional and vice versa. Professionals at some point in their careers make decisions based on professional conduct rather than on their own ethical ideals, simply because they are professional and act professionally. Still, this does not mean they are confident in their decisions and happy about the burden they bring. Rational arguments about the rightness of these decisions do not satisfy Schweitzer since in this situation the professional acts against her/his own will (e.g. performing triage or deciding how to divide up material resources). It is hard to find the answers to these dilemmas in Schweitzer's work and this is one of the weaknesses in his ethics.

Producing external norms for professionals during disasters is helpful for another reason. They provide a framework for quick and prompt decision making, fundamental to situations such as these. Is there time to show absolute reverence for all life in situations where you see suffering and pain at every corner? Of course, these norms cannot be understood in absolute terms (because of the unpredictability and complexity of disasters which often brings new problems yet to be considered $)^{11}$ and professionals must be prepared to combine external norms with their own thinking, ethics, and responsibility. Yet the ethical guidelines are important for disaster relief teams (and related professions) as they state the required minimum of professional ethical conduct (e.g. accepting cultural differences when helping in countries with different cultural and religious backgrounds).

Another problem arising from Schweitzer's understanding of ethics is his notion of responsibility. During disasters, there is often a need to track the responsibility of professional organizations or governmental bodies for the ethical decisions and actions they make. In the chaos of disaster, there is a need to establish order and the basic mechanism for relieving the community from the negative effects of the disaster (e.g. setting up camps, organizing disaster relief and health care teams, collecting resources, materials and food).

\footnotetext{
${ }^{11}$ This has been proved many times since the first ethical guidelines and norms for disaster relief teams were adapted (Leaning \& Guha-Sapir, 2013).
} 
Many decisions are made collectively. In these situations understanding responsibility simply in its pure individualist form as responsibility for all life may be difficult.

Despite these weak points in Schweitzer's understanding of ethics, there are some concepts which may inspire us and positively direct the discussion on disaster ethics. The first is Schweitzer's belief that people are capable of and willing to live their lives in such a way as to develop humanity. This positive attitude is necessary during disasters, as it offers us an ethical perspective in which the victims of disasters are seen through the scope of love, humanity, and solidarity. Importantly, Schweitzer does not leave this ethical will to the abstract or theoretical level. He believes it needs acting upon. These virtues and the ethical will motivate us to do good, to act. The suffering brought by disaster does not disappear on its own or through people feeling compassion for those suffering. It can be relieved only by the hard work of those who (either directly or indirectly) seek to remove it.

Secondly, Schweitzer understands that man was not born virtuous and that he has to morally develop throughout his life. Man's progress can be seen as an extension of this responsibility to life. On one hand, it may be difficult to fulfil this task (but not impossible) amidst all the dramatic events. On the other hand, it could be a useful concept for discussing disasters with outsiders. Raising awareness about humanitarian aid in Ukraine, about the need to show solidarity with refugees in Europe, or about the limits of health care in the Gaza Strip, can help individuals to see these people as a part of the world and humankind. A holistic sense of the individual's responsibility is the cornerstone of a modern and much needed understanding of humanism.

\section{Conclusion}

Albert Schweitzer may not have directly referred to natural disasters and catastrophes, such as floods or earthquakes, and he may not have described the professional conduct of a physician in an epidemic environment. Nonetheless, his focus on education and the moral development of individual human beings may provide us with a platform for discussing ethical decision making in disaster settings. The idea of humanism and man's main virtue (reverence for life) can be found throughout Albert Schweitzer's philosophical work and it presents us with an opportunity to analyse some of the features of virtue ethics and how (and if) they might enrich discussions on disaster ethics. Some of these concepts have been critically assessed in this paper, particularly the specific character of Schweitzer's understanding of ethics and its relationship to the contemporary world and civilisation.

\section{Acknowledgment}

I am grateful for feedback on an early version of this paper provided by participants at the workshop on moral theories and disasters at the University of Prešov, Slovakia (13-15 May 2015). Funding for the workshop on moral theories and disasters at the University of Prešov, Slovakia (13-15 May 2015) and open-access publication was provided by COST Action IS1201 (http://DisasterBioethics.eu). 


\section{References}

Bergel, K. (1946). Albert Schweitzer's reverence for life. The Humanist, 6(1), 31-34.

Cicovacki, P. (2009). Albert Schweitzer's ethical vision: A sourcebook. Oxford: Oxford University Press.

Cousin, N. (1960). Dr. Schweitzer of Lambaréné. New York: Harper \& Brothers.

Etkin, D., \& Leman Stefanovic, I. (2005). Mitigating natural disasters: The role of eco-ethics. Mitigation and Adaptation Strategies for Global Change, 10(3), 467-490.

Fromm, E. (1956). The art of loving. New York: Harper\&Row.

Funk, R. (2000). Erich Fromm. His life and ideas. New York: Continuum.

Hursthouse, R. (1995). Applying virtue ethics. In R. Hursthouse, G. Lawrence, \& W. Quinn (Eds.), Virtues and reasons (pp. 57-75). Wotton-under-Edge: Clarendon Press.

Kraus, O. (1944). Albert Schweitzer: His work and his philosophy. London: Adam \& Charles Black.

Leaning, J., \& Guha-Sapir, D. (2013). Natural disasters, armed conflict and public health. The New England Journal of Medicine, 369(19), 1836-1842.

Lepora, C. (2012). On compromise and being compromised. The Journal of Political Philosophy, 20(1), $1-22$.

Luegenbiehl, H.C. (2007). Disasters as object lessons in ethics: Hurricane Katrina. IEEE Technology and Society Magazine, Winter 2007, 10-15.

O’Mathúna, D. (2014). Disasters. In A.M.J. Henk, ten Have, \& B. Gordijn (Eds.), Handbook of global bioethics (pp. 619-639). Dortrecht: Springer.

Schweitzer, A. (1923). The philosophy of civilisation. Civilisation and ethics. Yale: Black.

Schweitzer, A. (1954). The problem of peace. Retrieved from http://www.nobelprize.org/nobel_prizes/ peace/laureates/1952/schweitzer-lecture.html

WHO (2002). Disasters \& Emergencies. Addis Ababa: WHO. Retrieved from http://apps.who.int/ disasters/repo/7656.pdf

Institute of Ethics and Bioethics,

Faculty of Arts,

University of Prešov,

17 Novembra 1 ,

SK-08078 Prešov,

Slovakia

E-mail: katarina.komenska@unipo.sk 